\title{
ON THE DEVELOPMENT OF A CONVERGENCE THEORY OF SYNTHESIS METHODS FOR SOLVING DIFFUSION EQUATIONS
}

\author{
Beny Neta and H. D. Victory, JR \\ Institute for Numerical Transport Theory, Department of Mathematics, \\ Texas Tech University, Lubbock, Texas 79409, U.S.A.
}

\begin{abstract}
This article is a survey of the theoretical questions arising in the development of a convergence theory and error analysis of synthesis methods for solving neutron dif fusion problems. For simplicity, we discuss convergence and the error analysis for spectral synthesis methods, in which the trial functions are functions solely of energy. The diffusion coefficient, the total and scattering cross-section data for the diffusion model are assumed spatially and energy dependent, and interior interfaces (i.e. spatial discontinuities in the diffusion coefficient and cross-section data) are present. The boundary conditions imposed are homogeneous Dirichlet conditions.
\end{abstract}

\section{KEYWORDS}

Neutron diffusion equation; spectral synthesis methods; finite elements; projection methods; Sobolev spaces.

\section{INTRODUCTION}

The need for establishing a theoretical foundation for synthesis methods for solving the neutron diffusion equation has been pointed out most concisely by R. Fröhlich (1972) who indicated that theoretical research is needed in the following areas: (i). Establishment of convergence results with accompanying error analysis; (ii). Development of systematic procedures for selecting test and trial functions. From the way synthesis methods are defined in which our approximations are sought in the

from $\sum_{i=1}^{N} w_{i}(\underline{x}) \psi_{i}(\underline{x}, y)$ where $\underline{x}$ and $\underline{y}$ are complementary subsets of the independent variables and $\psi_{i}$ and $w_{i}, i=1, \ldots, N$, are the known test functions and combining coefficients respectively, it is clear that many of the techniques of finite elements can be brought to shed light on these two areas. For one thing, the analysis can take place in a function space more appropriate for reflecting the qualitative behavior of solutions to the neutron diffusion equation, with boundary and interface conditions (e.g. the Sobolev-like space W of Meyer and Nelson (1979)) than the 
$\mathrm{L}^{2}$ setting in an earlier work of these authors (1977); secondly and more importantly, finite element analysis can give results on orders of convergence of the synthesis approximants, which can perhaps suggest techniques for addressing the second area of endeavor outlined by Fröhlich.

In this survey article, we shall discuss the considerations in the preceding paragraph for spectral synthesis methods for ascertaining energy dependence of solutions to neutron diffusion problems. Here the trial functions are functions solely of energy. The neutron diffusion model is quite general: the diffusion coefficient, total and scattering cross-section data are energy dependent; and interfaces spatial discontinuities in the diffusion coefficient and cross-section data - are assumed present. We shall address first the problem of ascertaining existence and uniqueness of solutions to a weak formulation of the neutron diffusion equation in which homogeneous Dirichlet boundary conditions are imposed, and then the approximation theoretic questions. Throughout the discussion, we shall point out open questions where research needs to be done, and is being done by the authors.

\section{THE ENERGY-DEPENDENT NEUTRON DIFFUSION PROBLEM}

Let $\nabla$ be the gradient operator in the space variable $\underline{x}=\left(x_{1}, \ldots, x_{m}\right) \in \Omega \subset R^{m}$ $(\mathrm{m}=1,2$, or 3 ) and let $E \in[0, \infty)$ represent energy. Take $D(\underline{x}, E)$ to be the diffusion coefficient; $\Sigma(\underline{x}, E)$, the total cross-section; $S(\underline{x}, E)$, the spontaneous source; and $\Phi(\underline{x}, E)$, the neutron density. Here, $D$ and $S$ are at the very least nonnegative, Lebesgue measurable functions on $\Omega \times[0, \infty) ; \Sigma$ is a positive, measurable function on the same region; and $k$ is a nonnegative measurable function on $\Omega \times[0, \infty) \times[0, \infty)$.

The region $\Omega$ is taken to be bounded and open. Further, we assume that $\Omega$ is (possibly) divided into a finite number of subdomains $\Omega_{i}$, each with boundary $\partial \Omega_{i}$. Each subdomain $\Omega_{i}$ is locally Lipschitzian, i.e. each point $x_{0} \in \partial \Omega_{i}$ has a neighborhood $\mathrm{U}\left(\mathrm{x}_{0}\right)$ such that $\partial \Omega_{i} \cap \mathrm{U}\left(\mathrm{x}_{0}\right)$ is the graph of a Lipschitz-continuous function. Portions of the $\partial \Omega_{i}$ will make up the interfaces between the regions $\Omega_{i}$, and the rest will make up the boundary $\partial \Omega$ of $\Omega$.

The classical diffusion problem we discuss is: Find a suitably smooth $\Phi$ such that

$$
\begin{aligned}
& -\nabla \cdot[D(\underline{\mathrm{x}}, \mathrm{E}) \nabla \Phi(\underline{\mathrm{x}}, \mathrm{E})]+\Sigma(\underline{\mathrm{x}}, \mathrm{E}) \Phi(\underline{\mathrm{x}}, \mathrm{E})- \\
& \int_{0}^{\infty} \mathrm{k}\left(\underline{\mathrm{x}}, \mathrm{E}, E^{\prime}\right) \sum\left(\underline{\mathrm{x}}, \mathrm{E}^{\prime}\right) \Phi\left(\underline{\mathrm{x}}, \underline{E}^{\prime}\right) \mathrm{d} E^{\prime}=\mathrm{S}(\underline{\mathrm{x}}, \mathrm{E}),(\underline{\mathrm{x}}, \mathrm{E}) \in \Omega \times[0, \infty) \text {; } \\
& \Phi(\underline{x}, E)=0 \quad, \quad(\underline{x}, E) \in \partial \Omega \times[0, \infty)
\end{aligned}
$$

$\Phi(\underline{x}, E)$ and $\frac{\partial \Phi}{\partial \nu}(\underline{x}, E)$ are continuous across interfaces in $\Omega$, where $\frac{\partial}{\partial v}$ denotes differentiation in the outward normal direction.

We consider existence and uniqueness questions for solutions to a weak formulation of (1) In the following Hilbert space, $\mathrm{H}_{0}^{1}\left(\Omega, \mathrm{L}^{2}[0, \infty)\right)$, consisting of functions $\psi$ such that $\underline{x} \rightarrow \psi(\underline{x}, \cdot)$ and $\underline{x} \rightarrow \frac{\partial \psi}{\partial x_{i}}(\underline{x}, \cdot)$ are $L^{2}$-mappings of $\Omega$ to $L^{2}[0, \infty)$ and endowed with the norm,

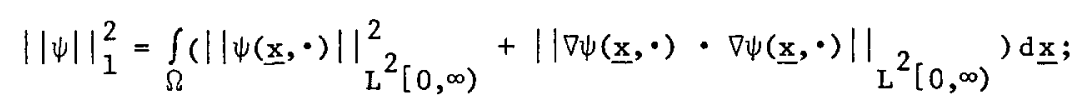


in the topology generated by $(2), \mathrm{H}_{0}^{1}\left(\Omega, \mathrm{L}^{2}[0, \infty)\right)$ is the completion of $\mathrm{C}_{0}^{\infty}\left(\Omega, \mathrm{L}^{2}[0, \infty)\right)$, the set of $\mathrm{C}^{\infty}$-functions having compact support with range in $\mathrm{L}^{2}[0, \infty)$. We remark that this Sobolev space of vector-valued functions is the space utilized by Nelson and Meyer (1979), and more recently by Neta (1981), in their formulation of a weak version of (1).

The following notation for inner products and norms will be used: For any $u, v \in$ $\mathrm{H}_{0}^{1}\left(\Omega, \mathrm{L}^{2}[0, \infty)\right)$,

$$
\begin{gathered}
(\mathrm{u}, \mathrm{v})=\int_{\Omega} \int_{0}^{\infty} \mathrm{u}(\underline{\mathrm{x}}, \mathrm{E}) \mathrm{v}(\underline{\mathrm{x}}, \mathrm{E}) \mathrm{dEd} \underline{\mathrm{x}}, \\
(\nabla \mathrm{u}, \nabla \mathrm{v})=\int_{\Omega} \int_{0}^{\infty} \nabla \mathrm{u}(\underline{\mathrm{x}}, \mathrm{E}) \cdot \nabla \mathrm{v}(\underline{\mathrm{x}}, \mathrm{E}) \mathrm{dEd} \underline{\mathrm{x}}, \\
\langle\mathrm{u}, \mathrm{v}\rangle=(\mathrm{u}, \mathrm{v})+(\nabla \mathrm{u}, \nabla \mathrm{v}), \\
|| \mathrm{u} \|_{0}=(\mathrm{u}, \mathrm{u})^{1 / 2}\left(\mathrm{~L}_{2} \text {-norm }\right),^{1 / 2}{ }_{\left(\mathrm{H}_{0}^{1} \text {-norm }\right) .}
\end{gathered}
$$

To formulate a weak version of $(1)$, we define the operators $\underset{\sim}{K}, \underset{\sim}{L}$ by

$$
\begin{aligned}
& \underset{\sim}{\mathrm{Ku}}(\underline{\mathrm{x}}, \mathrm{E})=\int_{0}^{\infty} \mathrm{k}\left(\underline{\mathrm{x}}, \mathrm{E}, \mathrm{E}^{\prime}\right) \Sigma\left(\underline{\mathrm{x}}, \mathrm{E}^{\prime}\right) \mathrm{u}\left(\underline{\mathrm{x}}, \mathrm{E}^{\prime}\right) \mathrm{d} \mathrm{E}^{\prime}, \\
& \underset{\sim}{\mathrm{Lu}}(\underline{\mathrm{x}}, \mathrm{E})=-\nabla \cdot[\mathrm{D}(\underline{\mathrm{x}}, \mathrm{E}) \nabla \mathrm{u}(\underline{\mathrm{x}}, \mathrm{E})]+\Sigma(\underline{\mathrm{x}}, \mathrm{E}) \mathrm{u}(\underline{\mathrm{x}}, \mathrm{E})-\underset{\sim}{\mathrm{Ku}}(\underline{\mathrm{x}}, \mathrm{E}),
\end{aligned}
$$

where $u$, resides, say, in $C_{0}^{\infty}\left(\Omega, L^{2}[0, \infty)\right)$ for the differentiations in the operator $L_{\sim}$ to be defined in a "classical" sense. The bilinear form $B(u, v)$ associated with (1), generated by assuming $u$ is a suitably smooth solution of (1) and $v$ smooth, is

$$
B(u, v)=(D \nabla u, \nabla v)+\left(\sum u, v\right)-(\underset{\sim}{K u}, v),
$$

and makes sense for $u, v \in H_{0}^{1}\left(\Omega, L^{2}[0, \infty)\right)$, when $\underset{\sim}{K}$ is a mapping of $H_{0}^{1}\left(\Omega, L^{2}[0, \infty)\right)$ to $\mathrm{L}^{2}(\Omega \times[\overline{0}, \infty))$. The variational formulation of the "classical" diffusion problem is: Find $\Phi \in \mathrm{H}_{0}^{1}\left(\Omega, \mathrm{L}^{2}[0, \infty)\right)$ such that

$$
B(\Phi, v)=(S, v) \text {, for all } v \in H_{0}^{1}\left(\Omega, L^{2}[0, \infty)\right)
$$

with $S(x, E) \in L^{2}(\Omega \times[0, \infty))$.

Nelson and Meyer (1979) showed existence and uniqueness of solutions to (6) in $\mathrm{H}_{0}^{1}\left(\Omega, \mathrm{L}^{2}[0, \infty)\right)$ by imposing the following conditions involving $\mathrm{D}(\mathbf{x}, \mathrm{E}), \Sigma(\underline{x}, \mathrm{E})$, and $k\left(\underline{x}, E, E^{\prime}\right)$ : 


$$
\begin{aligned}
& (D \nabla u, \nabla u) \geq c_{1}|| \nabla u||_{0}^{2}, \\
& |(D \nabla \mathrm{u}, \nabla \mathrm{v})| \leq\left. c_{2}|| \mathrm{u}\left|\|_{1}\right||\mathrm{v}|\right|_{1} \text {, } \\
& |(\Sigma \mathrm{u}+\underset{\sim}{\mathrm{K}} \mathrm{u}, \mathrm{v})| \leq \mathrm{c}_{3}|| \mathrm{u}\left\|_{0}|| \mathrm{v}\right\|_{0}, \\
& (\Sigma \mathrm{u}-\underset{\sim}{\mathrm{K}} \mathrm{u}, \mathrm{u}) \geq\left.\mathrm{c}_{4}|| \mathrm{u}\right|_{0} ^{2},
\end{aligned}
$$

where $c_{1}, c_{2}, c_{3}$, and $c_{4}$ are positive constants. Condition (7d) is undoubtedly the most restrictive condition - roughly speaking (7d) implies that the "adsorption operator", $(\Sigma \cdot-\underset{\sim}{K} \cdot)$, is invertible on $L^{2}(\Omega \times[0, \infty))$. Together, these conditions imply that the operator $\mathrm{L}$ is $\mathrm{H}_{0}^{1}\left(\Omega, \mathrm{L}^{2}[0, \infty)\right.$ ) - elliptic (Aubin(1972)), and existence and uniqueness are easy consequences of the Lax-Milgram Lemma (Bers, John, Schechter (1964)). Though condition (7d) is used to show that (6) is subcritical (in the sense that (6) has a unique solution in $\mathrm{H}_{0}^{1}$ ), an example provided by Meyer and Nelson (1979) shows that there are submultiplying situations where (7d) is violated. We now turn to discussing some ideas for weakening (7d).

In the following, we sha11 retain condition ( $7 a$ ), but replace conditions (7b-d) by the following:

$$
\begin{array}{r}
(D \nabla u, \nabla v)+(\Sigma u, v) \leq c_{2}|| u\left\|_{1}|| v\right\|_{1} ; \\
|| \underset{\sim}{\mathrm{k}} \|_{2}^{2}=\operatorname{Sup}_{\underline{x} \in \bar{\Omega}} \int_{0}^{\infty} \int_{0}^{\infty} \mathrm{k}^{2}\left(\underline{x}, \mathrm{E}^{2}, \mathrm{E}^{\prime}\right) \Sigma^{2}\left(\underline{\mathrm{x}}, \mathrm{E}^{\prime}\right) \mathrm{d} \mathrm{E}^{\prime} \mathrm{dE}<\infty \quad ;
\end{array}
$$

$$
\sup _{\underline{x} \in \bar{\Omega}} \int_{0}^{\infty} \int_{0}^{\infty}\left|k\left(\underline{x}+\underline{y}, E+t, E^{\prime}\right) \sum\left(\underline{x}+\underline{y}, E^{\prime}\right)-k\left(\underline{x}, E^{\prime}, E^{\prime}\right) \sum\left(\underline{x}, E^{\prime}\right)\right|^{2} d E^{\prime} d E \rightarrow 0
$$

as $|y|_{2}+|t| \rightarrow 0$, where $|y|_{2}$ is the Euclidean norm in $R^{\mathrm{m}}$,

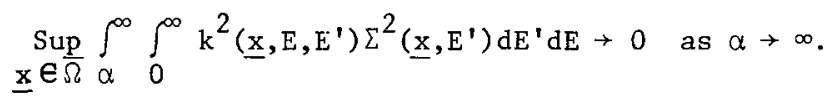

With these conditions, we can show that the operator A consisting of the diffusion term and the collision removal term, and given by

$$
\underset{\sim}{A} \cdot:=-\nabla \cdot(D(\underline{x}, E) \nabla \cdot)+\sum(\underline{x}, E) \cdot,
$$

can be shown to be a continuous isomorphism of $\mathrm{H}_{0}^{1}\left(\Omega, \mathrm{L}^{2}[0, \infty)\right.$ ) to its dual, $\left(\mathrm{H}_{0}^{1}\left(\Omega, \mathrm{L}^{2}[0, \infty)\right)\right)^{*}$ by virtue of the continuity of the associated bilinear form,

$$
\mathrm{a}(\mathrm{u}, \mathrm{v})=(\mathrm{D} \nabla \mathrm{u}, \nabla \mathrm{v})+(\Sigma \mathrm{u}, \mathrm{v}),
$$

on $\mathrm{H}_{0}^{1}\left(\Omega, \mathrm{L}^{2}[0, \infty)\right) \times \mathrm{H}_{0}^{1}\left(\Omega, \mathrm{L}^{2}[0, \infty)\right)$, due to $\left(7 \mathrm{~b}^{\prime}\right)$. More precisely, we can show 
Lemma 1: The operator $\underset{\sim}{\mathrm{A}}$ is $\mathrm{H}_{0}^{1}\left(\Omega, \mathrm{L}^{2}[0, \infty)\right)$-elliptic, i.e. there is a positive constant $c_{1}$ such that $a(u, u) \geq c_{1}|| u \|_{1}{ }^{2}$, from which we conclude that $\underset{\sim}{A}$ is continuous isomorphism of $\mathrm{H}_{0}^{1}\left(\Omega, \mathrm{L}^{2}[0, \infty)\right)$ to its dual.//

The conditions ( $\left.7 c^{\prime}-d^{\prime}\right)$ enable us to show that $\underset{N}{K}$ in $(4 a)$ is a compact mapping from $\mathrm{H}_{0}^{1}\left(\Omega, \mathrm{L}^{2}[0, \infty)\right)$ to $\mathrm{L}^{2}(\Omega \times[0, \infty))$; the fact that $\mathrm{H}_{0}^{1}\left(\Omega, \mathrm{L}^{2}[0, \infty)\right)$ can be continuously imbedded into $\mathrm{L}^{2}(\Omega \times[0, \infty))$, which in turn can be continuously imbedded into the dual of $\mathrm{H}_{0}^{1}\left(\Omega, \mathrm{L}^{2}[0, \infty)\right)$, allows us to infer that $\underset{\sim}{\mathrm{L}}$ is a continuous mapping from $\mathrm{H}_{0}^{1}\left(\Omega, \mathrm{L}^{2}[0, \infty)\right)$ to its dual. The proofs of these facts are relegated to a technical report by Neta and Victory, and we refer the interested reader to this item in the References.

It is well known by the Riesz-Schauder Theory (Aubin(1972)) that the operator $\underset{\sim}{\mathrm{A}}+\lambda \underset{\sim}{\mathrm{K}}$ is either invertible, or else it and its adjoint have closed ranges, and finite and equal - dimensional nullspaces. In the latter case, $\lambda$ is an element of "the spectrum of $\mathrm{K}_{\sim}$ relative to $\mathrm{\sim}^{\prime \prime}$ (Aubin(1972)). We assume that the problem (6) is subcritical in that 1 is not a spectral point. To summarize, we have

Theorem 1: Under subcritical conditions(in which 1 is not in the spectrum of $\mathrm{K}$ relative to $\mathrm{A}$ ), the weak formulation of the classical diffusion problem described by (6) has a unique solution $\Phi \in \mathrm{H}_{0}^{1}\left(\Omega, \mathrm{L}^{2}[0, \infty)\right) . / /$

In the next section, we shall study the convergence properties of spectral synthesis approximations to the solution to a weak formulation in (6) of a subcritical neutron diffusion problem. But first, we shall note some further questions concerning existence and uniqueness of solutions to the classical formulation of the diffusion problem in (1). What comes to mind first of all is whether the weak formulation in (6) will in turn yield "classical" solutions of (1) under appropriate smoothness assumptions on $\Sigma(\underline{x}, E), k\left(\underline{x}, E, E^{\prime}\right), D(\underline{x}, E)$, and $S(\underline{x}, E)$. By a "classical" solution we mean a function $\Phi \in \mathrm{C}^{2}\left(\Omega, \mathrm{L}^{2}[0, \infty)\right.$ ) (twice-differentiable functions with range in $L^{2}[0, \infty)$ ) such that (i) $\Phi(\underline{x}, \cdot)=0$ a.e. in energy $E, \underline{x} \in d \Omega$; (ii) $\Phi(\underline{x}, \cdot)$ and $\mathrm{D}(\underline{\mathrm{x}}, \cdot) \frac{\partial \Phi}{\partial \nu}(\underline{\mathrm{x}}, \cdot)$ are continuous mappings into $\mathrm{L}^{2}[0, \infty)$ as $\underline{\mathrm{x}}$ goes across interfaces in $\Omega$.

Towards addressing this question, the authors for an initial study are currently developing the existence and uniqueness analysis to neutron diffusion problems for one spatial dimension, with mixed Dirichlet and Neumann boundary conditions. The work is in a preliminary stage at this point, but the work by Aubin (1972, pp. 210$212,252-254)$ on interface elliptic boundary-value problems has potential for being generalized to our setting. Technical problems for the complete rigorous analysis include, for example, developing a Trace Theory for Sobolev spaces of vector-valued functions, but this task appears to be a straight-forward generalization of the classical theory in Aubin (1972, pp. 189-204).

For higher spatial dimensions, the possibility of intersecting interfaces complicates the analysis considerably, as is well-known. As pointed out by Kellogg (1971), in such a higher-dimensional setting, complications can arise at "singular points" of the domain $\Omega$ or subdomains $\Omega_{i}$, i.e. those points where two interface curves or surfaces cross, where an interface curve or surface meets the boundary of $\Omega$, or where an interface or boundary has a discontinuous tangent (a corner). At this point, it is clear that an analysis for obtaining classical solutions from a weak formulation should be built upon the work of Kellogg (1971) on interface problems and on the 
fundamental work of Kondrat'ev (1968) concerning smoothness of solutions to elliptic boundary-value problems near conical or angular points. To recover the interface constraints for a classical solution from the variational formulation, perhaps a Lagrange multiplier variational formulation such as studied by Babuska (1970 a,b; 1973) for elliptic boundary value problems could be very useful in this regard; also Lagrange multipliers may allow us to use spatially discontinuous trial functions in general synthesis methods. Such classes of functions have, for example, been used by Buslik $(1966 \mathrm{a}, \mathrm{b})$. These topics and ideas are a part of the spectrum of research into developing a convergence theory for synthesis methods.

\section{CONVERGENCE RESULTS AND ERROR ESTIMATES}

To specify the synthesis method, we first prescribe a set $\left\{\psi_{i}(E): E \in[0, \infty), i=1\right.$, $2, \ldots, N\}$ in $L^{2}[0, \infty)$. These functions define a subspace $R_{N} \subset H_{0}^{1}\left(\Omega, L^{2}[0, \infty)\right.$ ) given by

$\mathrm{R}_{N}=\left\{\Psi \in \mathrm{H}_{0}^{1}\left(\Omega, \mathrm{L}^{2}[0, \infty)\right): \Psi(\underline{x}, \mathrm{E})=\sum_{i=1}^{N} \mathrm{w}_{i}(\underline{x}) \Psi_{i}(E), w_{i}(\underline{x}) \in H_{0}^{1}(\Omega), i=1,2, \ldots, N\right\}$,

where $H_{0}^{1}(\Omega)$ denotes the usual Sobolev-space of real valued functions $\psi$ having zero trace on $\partial \Omega$ such that $\psi$ and $\frac{\partial \psi}{\partial x_{i}}$ lie in $L^{2}(\Omega)$. Clearly, with respect to the topology of $\mathrm{H}_{0}^{1}\left(\Omega, \mathrm{L}^{2}[0, \infty)\right), \mathrm{R}_{\mathrm{N}}$ is closed in $\mathrm{H}_{0}^{1}\left(\Omega, \mathrm{L}^{2}[0, \infty)\right)$ and is thereby a Hilbert space. Our

fundamental assumption for the convergence analysis is that the sequence $\left\{R_{N}\right\}$ is complete in $\mathrm{H}_{0}^{1}\left(\Omega, \mathrm{L}^{2}[0, \infty)\right)$, i.e. to each $\mathrm{f} \in \mathrm{H}_{0}^{1}\left(\Omega, \mathrm{L}^{2}[0, \infty)\right)$, there is a sequence $\left\{\mathrm{f}_{\mathrm{N}}\right\}$, with $\mathrm{f}_{\mathrm{N}} \in \mathrm{R}_{\mathrm{N}}$ such that $\mathrm{f}_{\mathrm{N}} \rightarrow \mathrm{f}$.

We shall view spectral synthesis mehtods as projection methods, and our analysis will use some fundamental results as projection methods discussed by witsch (1977). The spectral synthesis approximations are determined by finding $\Phi \in R_{\mathrm{N}}$ such that

$$
\mathrm{B}(\Phi, \Psi)=(\mathrm{S}, \Psi) \text {, for a11 } \Psi \in \mathrm{R}_{\mathrm{N}}, \mathrm{S} \in \mathrm{L}^{2}(\Omega \times[0, \infty)) \text {. }
$$

By definition of $B$, however, we view this problem as of finding $\Phi \in R_{N}$ such that

$$
((\underset{\sim}{\mathrm{A}}-\underset{\sim}{\mathrm{K}}) \Phi, \Psi)\left(\mathrm{H}_{0}^{1}\right) * \times \mathrm{H}_{0}^{1}=(\mathrm{S}, \Psi)\left(\mathrm{H}_{0}^{1}\right)^{*} \times \mathrm{H}_{0}^{1}
$$

for all $\Psi \in \mathrm{R}_{\mathrm{N}}$, with $(\underset{\sim}{\mathrm{A}}-\underset{\sim}{\mathrm{K}}) \Phi$ and $\mathrm{S}$ considered as elements of $\left(\mathrm{H}_{0}^{1}\left(\Omega, \mathrm{L}^{2}[0, \infty)\right)\right)^{*}$, and $(\cdot, \cdot)\left(\mathrm{H}_{0}^{1}\right)^{*} \times \mathrm{H}_{0}^{1}$ denoting the duality pairing on $\left(\mathrm{H}_{0}^{1}\left(\Omega, \mathrm{L}^{2}[0, \infty)\right)\right)^{*} \times \mathrm{H}_{0}^{1}\left(\Omega, \mathrm{L}^{2}[0, \infty)\right)$.

Our subcriticality hypotheses assume that $(A-\underset{\sim}{(})$ is invertible. We now state a fundamental result concerning convergence of projection methods for operators compactly perturbed:

Theorem (Witsch (1977)): Let a projection method for L be given, and suppose $\underset{\sim}{A}=\underset{\sim}{\mathrm{L}}+\underset{\sim}{\mathrm{is}}$ an invertible mapping of the domain space ${ }^{\sim} \mathrm{R}$ to the range space $T$. Also, suppose $\underset{\sim}{-1} \underset{\sim}{C}$ is a compact operator in $R$. 


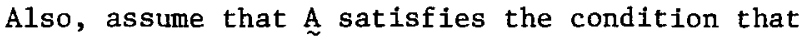

$$
\text { ( } \left.u \in R_{\mathrm{N}},(\mathrm{v}, \underset{\sim}{\mathrm{Au}})=0 \mathrm{Vv} \in \mathrm{T}_{\mathrm{N}}^{*}\right) \Rightarrow \mathrm{u}=0
$$

(where $T_{N}{ }^{*}$, the space of "test functions," is a finite dimensional subspace of the dual $T^{*}$ to $T$, and $(\cdot, \cdot)$ is the duality pairing on $\left.T \times T^{*}\right)$. Suppose the projection operators ${\underset{\sim}{0}}_{N}$ constructed for $\underset{\sim}{A}$ are uniform1y bounded, where the $\left\{\tilde{Q}_{N}\right\}$ map $R_{N} \rightarrow R$ and are defined by $\tilde{\mathrm{Q}}_{\mathrm{N}} \mathrm{u}^{*}=\mathrm{u}_{\mathrm{N}}$ with $\mathrm{u}^{*}$ and $\mathrm{u}_{\mathrm{N}}$ solutions to exact and approximate problems respectively. Also, let the sequence $\left\{R_{N}\right\}$ be complete in $R$.

Then the ${\underset{\sim}{N}}_{\mathrm{N}}$ constructed for $\mathrm{L}_{\sim}$ are defined for sufficiently large $N$ and are uniformly bounded. Hence, $\mathrm{u}_{\mathrm{N}} \rightarrow \mathrm{u}^{*}={\underset{\sim}{\mathrm{L}}}^{-1} \mathrm{f}$, for each $\mathrm{f}$ in the range space $\mathrm{T} . / /$

We remark that a detailed discussion of the existence of the projection operators $\left\{\mathrm{Q}_{\mathrm{N}}\right\}$ can be found in Witsch (1977). The essential point is that the ${\left\{\mathrm{Q}_{\mathrm{N}}\right.}_{\mathrm{N}}\}$ engendered by a projection method have a profound bearing on the convergence properties of $\left\{u_{N}\right\}$ - indeed if the $\left\{Q_{N}\right\}$ are uniformly bounded, optimal rates of convergence for $\left\{u_{N}\right\}$ result, where the error in approximating $u^{*}$ by $u_{N}$ is directly proportional to the error in the best approximation to $u^{*}$ from $R_{N}$ (cf. Theorem 2.2 of Witsch (1977)).

To apply the above theorem, we have in our setting that $\underset{\sim}{\mathcal{C}}$ is the scattering operator; $\underset{\sim}{A}$ the operator consisting of the diffusion term and the collision removal term with the spaces $\mathrm{R}$ and $\mathrm{T}$ denoting $\mathrm{H}_{0}^{1}\left(\Omega, \mathrm{L}^{2}[0, \infty)\right)$ and $\left(\mathrm{H}_{0}^{1}\left(\Omega, \mathrm{L}^{2}[0, \infty)\right)\right)^{*}$ respectively. The spectral synthesis approximation scheme for the neutron diffusion problem in ( 1 ) can be seen to effect a projection method for approximating solutions to $\underset{\sim}{A} \Phi=S$ via the following:

Find $\Phi \in \mathrm{R}_{\mathrm{N}}$ such that

$$
\mathrm{a}(\Phi, \Psi)=(\mathrm{S}, \Psi), \mathrm{V} \Psi \in \mathrm{H}_{0}^{1}\left(\Omega, \mathrm{L}^{2}[0, \infty)\right),
$$

where $S \in L^{2}(\Omega \times[0, \infty))$. As shown by Neta and Victory (1981), the fact that the operator $\underset{\sim}{\mathrm{A}}$ is $\mathrm{H}_{0}^{1}\left(\Omega, \mathrm{L}^{2}[0, \infty)\right)$ - elliptic by Lemma 1 , implies that $\underset{\sim}{\mathrm{A}}{ }^{-1}$ is continuous and, more importantly, that $\left\{\tilde{\mathrm{Q}}_{\mathrm{N}}\right\}$ are uniformly bounded. Hence the aforementioned theorem of $\mathrm{K}$. Witsch applies, and we have our fundamental result for convergence of the spectral synthesis approximations.

Theorem 2: If the diffusion problem (6) is subcritical, then the sequence of spectral synthesis approximations $\Phi_{\mathrm{N}}$ converge to the solution $\Phi$ of $(6)$ in the space $\mathrm{H}_{0}^{1}\left(\Omega, \mathrm{L}^{2}[0, \infty)\right)$, and, moreover,

$$
\left\|\Phi-\Phi_{N}\right\|_{1} \leq C_{3}\left\|\Phi-\psi_{N}\right\|_{1} \text {, }
$$

where $\psi_{\mathrm{N}}$ is any element of $\mathrm{R}_{\mathrm{N}}$, and where $\mathrm{C}_{3}$ is a constant equal to an upper bound for $\left(1+\|\left.\right|_{N} \mid\right)$, with $\left\{Q_{N}\right\}$, the uniformly bounded sequences of projection operators associated with $\underset{\sim}{\mathrm{L}}$ described in the above theorem of Witsch.// 
We remark that a complete sequence of subspaces could be constructed, for example, in the following way: Let $\left\{\psi_{j}(E), j=1,2, \ldots\right\}$ be a complete orthonormal sequence in $\mathrm{L}^{2}[0, \infty)$, and take $\left\{\theta_{j}(\underline{x}), j=1, \ldots\right\}$ to be any orthonormal sequence in $H_{0}^{1}(\Omega)$ (such a sequence exists because $\mathrm{H}_{0}^{1}(\Omega)$ is complete and separable). Then the subspace $\mathrm{R}_{\mathrm{N}}$ consists of functions $\Psi$ such that $\Psi$ is expressible in the form

$$
\Psi(\underline{x}, E)=\sum_{\substack{j+k \leq 2 N \\ k \leq N}} a_{j k}{ }^{\theta} j \underline{(x)} \psi_{k}(E) .
$$

From such subspaces, we extract our converging spectral synthesis approximations. Approximating subspaces considered by Meyer and Nelson $(1977,1979)$ are of this type.

A natural step to be takan in our convergence analysis is the study of rates of convergence, both in the $\mathrm{H}_{0}^{1}\left(\Omega, \mathrm{L}^{2}[0, \infty)\right)$ and $\mathrm{L}^{2}(\Omega \times[0, \infty))$ norms, of $\Phi_{\mathrm{N}} \in \mathrm{R}_{\mathrm{N}}$ to $\Phi \in \mathrm{R}$ solving (6). Ascertaining sharp rates of convergence will require some finer conditions to be imposed on $\mathrm{R}_{\mathrm{N}}$ than discussed in the preceding paragraph. What we have in mind is that our finite-dimensional-like subspaces satisfy a property of the following type for traditional finite-element spaces in the treatise by Strang and Fix ((1973, Chapter 3):

Approximation Property: There is an integer $k \geq 2$ and constants $c_{4}, c_{5}$ independent of $h$, such that for all $u$ in $H_{0}^{k}\left(\Omega, L^{2}[0, \infty)\right)$, there is a $v \in R_{N}$ such that

$$
|| \mathrm{u}-\mathrm{v}\left\|_{\ell} \leq \mathrm{c}_{4+\ell} \mathrm{h}^{\mathrm{k}-\ell}|| \mathrm{u}\right\|_{\mathrm{k}}, \ell=0,1,
$$

where

$$
|| \mathrm{u}||_{\mathrm{k}}=\left\{\int_{\Omega} \int_{0}^{\infty} \sum_{|\alpha| \leq \mathrm{k}}\left|\mathrm{D}_{\mathrm{x}}^{\alpha} \mathrm{u}(\underline{\mathrm{x}}, \mathrm{E})\right|^{2} \mathrm{dEd} \underline{\mathrm{x}}\right\}^{\frac{1}{2}} .
$$

and $\quad h=(1 / N)^{1 / m}, N=\operatorname{dim} R_{N}$.

From Theorem 2, we have immediately the following result:

Theorem 3: Let us suppose that $\Phi$ is a solution of (6) lying in $\mathrm{H}_{0}^{\mathrm{k}}\left(\Omega, \mathrm{L}^{2}[0, \infty)\right)$. Then we obtain that the spectral synthesis approximations $\Phi_{\mathrm{N}}$ converge to $\Phi$ with rate provided by

$$
\left\|\Phi-\Phi_{N}\right\|_{1} \leq \mathrm{Ch}^{\mathrm{k}-1}|| \Phi \|_{\mathrm{k}} \cdot / /
$$

To obtain estimates in the $\mathrm{L}^{2}$-norm, we first cite a lemma proved by Neta (1981): Lemma 2: Let $L^{*}$ be the adjoint operator to $L_{\sim}$. Let $B^{*}(u, v)$ be the bilinear form associated with $\stackrel{\sim}{*}_{\sim}^{*}$. Then

$$
B *(u, v)=B(v, u)
$$


and there exists a positive constant $c_{6}$ such that

$$
|| \Phi-\Phi_{N}\left\|_{0}^{2} \leq c_{6}|| w-z\right\|_{1}\left\|\Phi-\Phi_{N}\right\|_{1}
$$

where $\Phi$ is the solution of (6); $\Phi_{N}$, the solution of (11), and $w$ is the solution of

$$
B^{*}(w, v)=\left(\Phi-\Phi_{N}, v\right), \text { for a11 } v \in H_{0}^{1}\left(\Omega, L^{2}[0, \infty)\right) \text {, }
$$

with $z$ the best approximation to w from $\mathrm{R}_{\mathrm{N}} \cdot / /$

The following estimate in the $\mathrm{L}^{2}$-norm is immediate:

Theorem 4: Let $\Phi, \Phi_{N}$, w be as in Lemma 2. Then there is a positive constant $\lambda$ such that

$$
\left\|\Phi-\Phi_{N}\right\|_{0} \leq \lambda\|w-z\|_{1}^{1 / 2}|| \Phi-\psi_{N} \|_{1}^{\frac{1}{2}}
$$

where $\psi_{\mathrm{N}}$ is the best approximation to $\Phi$ from $\mathrm{R}_{\mathrm{N}} \cdot / /$

This result is not sharp, since the error in the $\mathrm{L}_{2}$-norm is not of the same order as that for the best approximation to $\Phi$ in the space $\mathrm{R}_{\mathrm{N}}$ with respect to the $\|\cdot\| \|_{1}-$ norm.

In order to obtain a sharper result, we need to assume that the solution w of (20) is regular, i.e.

$$
|| w\left\|_{2} \leq c_{7}\right\| \Phi-\Phi_{N} \|_{0}
$$

for some positive constant $c_{7}$. We get

Theorem 5: Let $\Phi$ and $\Phi_{N}$ be as before, and let w be a regular solution to the adjoint problem (20). Then

$$
\left\|\Phi-\Phi_{N}\right\|_{0} \leq h^{k}\left(c_{2} c_{5} c_{7}+c_{4} c_{7}|| \underset{\sim}{k} \|_{2} h\right)\|\Phi\|_{k} \cdot / /
$$

Proof: By (18) and $(20), B(v, w)=B^{*}(w, v)=\left(\Phi-\Phi_{N}, v\right)$ for all $v \in H_{0}^{1}\left(\Omega, L^{2}[0, \infty)\right)$. From the regularity of $\mathrm{w}$, one has

$$
|| w\left\|_{2} \leq c_{7}|| \Phi-\Phi_{N}\right\|_{0} .
$$

Let $\mathrm{v}=\Phi-\Phi_{\mathrm{N}} \in \mathrm{H}_{0}^{1}\left(\Omega, \mathrm{L}^{2}[0, \infty)\right)$; since $\mathrm{B}\left(\Phi-\Phi_{\mathrm{N}}, \mathrm{z}\right)=0$ for a11 $\mathrm{z} \in \mathrm{R}_{\mathrm{N}}$, we have

$$
\begin{gathered}
|| \Phi-\Phi_{N}||_{0}^{2}=B\left(\Phi-\Phi_{N}, w\right)=B\left(\Phi-\Phi_{N}, w-z\right) \\
\leq c_{2}|| w-z\left\|\left.\right|_{1}\right\| \Phi-\Phi_{N}\left\|_{1}+\right\| \underset{\sim}{k}\left\|_{2}|| w-z\right\|_{0}\left\|\Phi-\Phi_{N}\right\| \|_{0},
\end{gathered}
$$

with 


$$
\|\underset{\sim}{k}\|_{2}=\left(\operatorname{Sup}_{\underline{x} \in \bar{\Omega}} \int_{0}^{\infty} \int_{0}^{\infty} k^{2}\left(\underline{x}, E, E^{\prime}\right) d E^{\prime} d E\right)^{\frac{1}{2}} .
$$

By the approximation property, we have

$$
|| \Phi-\Phi_{N}||_{0}^{2} \leq c_{2} c_{5} h|| w||_{2}|| \Phi-\Phi_{N}\left|\left\|_{1}+\right\| \underset{\sim}{\alpha}\left\|{ }_{2} c_{4} h^{2}|| w||_{2}\right\| \Phi-\Phi_{N}\right| \|_{0} \text {. }
$$

Using (22), one has after division by $\left\|\Phi-\Phi_{N}\right\|_{0}$,

$$
\left\|\Phi-\Phi_{N}\right\|_{0} \leq c_{2} c_{5} c_{7} h|| \Phi-\Phi_{N}\left\|_{1}+c_{4} c_{7}|| \underset{\sim}{\mid k}\right\|_{2} h^{2}|| \Phi-\Phi_{N}\|\|_{0} .
$$

Combining (26) with (17), and using the inequality $\left\|\Phi-\Phi_{N}\right\|\left\|_{0} \leq\right\| \Phi-\Phi_{N}\|\|_{1}$, we get

$$
|| \Phi-\Phi_{N} \|_{0} \leq h^{k}\left(c_{2} c_{5} c_{7}+c_{4} c_{7}|| \underset{\sim}{K} \|_{2} h\right)|| \phi||_{k} .
$$

This completes the proof of Theorem 5 .

Remark: The regularity of $w$ enables us to pick up one more power of $h$, than appears in (17). We also remark that, for homogeneous media in which $D, \Sigma$, and $k$ are independent of the spatial variable $\underline{x}$, the $L^{2}$-behavior of $S(\underline{x}, E)$, along with the mapping properties of the scattering operator $\mathrm{K}$, implies that the solution of (6) would a-fortiori lie in $\mathrm{H}_{0}^{2}\left(\Omega, \mathrm{L}^{2}[0, \infty)\right)$. In the homogeneous setting, the variable $\mathrm{E}$ acts as a parameter, and regularity of $\Phi$ results from the analysis of Aubin (1972, Chapter 6) for the mapping properties of the Laplacian.

In problems, where we come to grips to ascertaining orders of convergence of spectral synthesis solutions, it is imperative that the precise regularity of solutions to (1) near singular points and interfaces be studied. The discussion concerning rates in the second half of this section has motivated research into such questions.

\section{ACKNOWLEDGMENT}

This research was supported by the U. S. National Science Foundation under NSF Grant No. CPE-800-7396.

\section{REFERENCES}

Aubin, J.P. (1972). Approximation of Elliptic Boundary-Value Problems. Wiley Interscience, New York.

Babuška, I. (1970a). Computing, 5, 207-213.

Babuška, I. (1970b). Computing, 6, 264-273.

Babuška, I. (1973). Numer. Math., 20, 179-192.

Bers, L., F. John, and M. Schechter (1964). Partial Differential Equations. John Wiley and Sons, New York.

Bus1ik, A.J. (1966). Trans. Am. Nuc1. Soc., 9, 199.

Buslik, A.J. (1966). A variational principle for the neutron-diffusion equation using discontinuous trial functions. WAPD-TM-610, Bettis Atomic Power Laboratory.

Frolich, R. (1972). Flux Synthesis Methods Versus Finite-Difference Approximation Methods for the Efficient Determination of Neutron Flux Distributions in Fast and Thermal Reactors. Proceedings of the IAEA Seminar on Numerical Reactor Calculations, 591-611 (Vienna). 
Kellogg, R.B. (1971). Numerical Solution of Partial Differential Equations II. B. Hubbard, Editor, 351-400.

Kondrat'ev, V.A. (1967). Trans. Moscow Math Soc. 16. (Am. Math. Soc. Trans1. (1968), 227-313).

Meyer, H.D., and P. Nelson (1979). ZAMP, 30, 901-912.

Nelson, P. and H.D. Meyer (1977). Nucl. Sci. Eng., 64, 638-643.

Neta, B. (1981). ZAMP. Accepted for publication.

Neta, B. and H.D. Victory, Jr. (1981). Convergence Results for Galerkin Spectral Synthesis Methods for Diffusion Equations with General Boundary Conditions. Institute for Numerical Transport Theory Technical Report (Texas Tech University). Strang, G., and G.J. Fix (1973). An Analysis of the Finite Element Method. PrenticeHal1, Inc., Englewood Cliffs, N.J.

Witsch, K. (1977). Numer. Math., 27, 339-354. 